\title{
MORBILIDAD Y MORTALIDAD NEONATAL ASOCIADA A LA DIABETES GESTACIONAL
}

\author{
Pablo Velázquez G. ${ }^{1}$, Genaro Vega M. PhD ${ }^{2}$, Martha Leticia Martínez M. PhD ${ }^{3}$ \\ 1 Programa de Medicina Familiar, Unidad de Medicina Familiar No. 16, Instituto Mexicano del Seguro Social, Delegación \\ Querétaro. ${ }^{2}$ Facultad de Medicina, Universidad Autónoma de Querétaro. ${ }^{3}$ Unidad de Medicina Familiar No. 13. Que- \\ rétaro, México.
}

\section{RESUMEN}

Objetivo: Determinar la asociación de la morbilidad y mortalidad neonatal con la diabetes gestacional. Método: Estudio tipo cohortes. Se realizó un estudio en embarazadas desde diciembre de 2007 a noviembre de 2008 en el servicio de tococirugía del Hospital General Regional No. 1 de Querétaro, México, se formaron dos grupos de 71 pacientes, uno con diabetes gestacional y otro sin ella. El muestreo fue por cuota pareado por edad. En ambos grupos se midieron variables sociodemográficas, antecedentes obstétricos, vía de interrupción del embarazo, morbilidad y mortalidad neonatal. Los resultados se analizaron con Chi cuadrado y riesgo relativo con un poder alfa de 0,05. Resultados: La tasa de morbilidad en el grupo expuesto fue de $60 \%$. Las variables que tuvieron significancia estadística fueron: la obesidad pregestacional (RR: 2,7), cesárea (RR: 3,3), complicaciones metabólicas (RR: 10), morbilidad respiratoria (RR: 6,7), macrosomía (RR: 4,1), hipoglucemia (RR: 14,2) y taquipnea transitoria del recién nacido (RR: 7,7). La edad materna, sobrepeso gestacional, nivel socioeconómico, escolaridad, antecedentes de macrosómicos, de cesáreas y de malformaciones congénitas; malformaciones congénitas, bajo peso neonatal, prematurez, enfermedad de membrana hialina, hiperbilirrubinemia, hipocalcemia, mortalidad neonatal y la asfixia no tuvieron asociación significativa. La complicación metabólica más frecuente fue: hipoglucemia $(17,2 \%)$. No hubo muertes perinatales. Conclusiones: El grupo expuesto estudiado mostró mayor morbilidad asociada a la diabetes gestacional que el grupo no expuesto, es necesario el diagnóstico temprano en mujeres con factores de riesgo para esta entidad y establecer un programa de tratamiento con vigilancia estrecha.

\section{PALABRAS CLAVE: Diabetes gestacional, morbilidad neonatal, mortalidad neonatal, riesgo perinatal}

\section{SUMMARY}

Objectives: To determine the association of neonatal morbidity and mortality with gestational diabetes. Methods: Study cohort. A study of pregnant women from December 2007 to November 2008 in the service of ginecology and obstetrics HGR No. 1 Queretaro, were divided into two groups of 71 patients, one gestational diabetic and one without it. The quota sampling was matched by age. In both groups were measured sociodemographic, obstetric history, via interruption of birth, neonatal morbidity and mortality. The results were analyzed with Chi square and relative risk with a power alpha of 0.05 . Results: The morbidity rate in the exposed group was $60 \%$. Variables that were statistically significant were: pregestational obesity (RR: 2.7), cesarean (RR 3.3), metabolic complications (RR 10), respiratory illness (RR: 6.7), macrosomia (RR: 4.1), hypoglycemia (RR: 14.2) and transient tachypnea of the newborn (RR: 7.7). Maternal age, gestational overweight, socioeconomic status, education, history of macrosomic, and cesarean birth defects, 
congenital malformations, low birth weight, prematurity, hyaline membrane disease, hyperbilirubinemia, hypocalcemia, and neonatal asphyxia had no significant association. The most common metabolic complication was hypoglycemia (17.2\%). There were no perinatal deaths. Conclusions: The exposed group showed higher morbidity associated with gestational diabetes that the unexposed group, early screening is necessary in women with risk factors for this disease and establish a treatment program with close monitoring.

\section{KEY WORDS: Gestational diabetes, neonatal morbidity, neonatal mortality, perinatal risk}

\section{INTRODUCCIÓN}

La diabetes gestacional (DG) es la complicación metabólica más frecuente del embarazo ya que afecta a más de $10 \%$ de las embarazadas mayores de 25 años (1). Se define por el diagnóstico de diabetes mellitus en el curso de un embarazo, ya sea por niveles de glucosa $>126 \mathrm{mg} / \mathrm{dl}$ en dos ocasiones o por tener una curva de tolerancia a la glucosa diagnóstica para diabetes mellitus gestacional (2). La diabetes gestacional representa el $90 \%$ de los casos de diabetes asociada al embarazo (3).

La prevalencia de la DG a nivel mundial oscila entre 2 a $9 \%$; en estudios realizados en México, corresponde al $4,3 \%$ encontrado en una población con derecho al seguro social, hasta $11 \%$ en poblaciones abiertas, dependiendo del criterio empleado para el diagnóstico (4).

La morbilidad y la mortalidad perinatal en las diabéticas se han convertido en un evento menos frecuente que en el pasado, debido a la implementación de programas educativos de detección precoz, tratamiento del trastorno metabólico y a la vigilancia fetal anteparto (5).

Los hijos de madres con diabetes gestacional tienen mayor riesgo de presentar complicaciones metabólicas asociadas; como la hipoglucemia con un riesgo del $20 \%$, prematurez $15 \%$, macrosomía $17 \%$, hiperbilirrubinemia $5,6 \%$, hipocalcemia $50 \%$, hipomagnesemia $50 \%$, síndrome de dificultad respiratoria $4,8 \%$, malformaciones congénitas de 5 a $12 \%$ o muerte neonatal, incrementando el riesgo hasta 5 veces más $(6,7)$.

Las grandes malformaciones congénitas (MC) siguen siendo la principal causa de mortalidad y morbilidad grave en lactantes hijos de mujeres con diabetes gestacional. La asociación de DM materna con anomalías congénitas es bien conocida, especialmente las mayores de los sistemas cardiovascular, nervioso central (SNC), genitourinario y esquelético (8).

En nuestro país la conducta y tratamiento de estas pacientes esta descrito en las normas nacionales y han posibilitado la disminución de las com- plicaciones usuales en las gestantes diabéticas. El objetivo del estudio es conocer la eficacia de estas normativas, a través del impacto de la asociación de la morbilidad y mortalidad neonatal con la diabetes gestacional.

\section{PACIENTES Y MÉTODO}

Diseño de la investigación. Se realizó un estudio de tipo cohortes, en embarazadas con y sin diabetes gestacional, usuarias del Hospital General Regional № 1 del IMSS, delegación Querétaro, de diciembre del 2007 a noviembre del 2008. Se calculó el tamaño de la muestra con el paquete estadístico Epi-Info 2002 con su fórmula para cohortes, con un $\mathrm{RR}$ de 1,5, factor de exposición del 30\% para los expuestos y del $10 \%$ para los no expuestos, poder $B$ del $80 \%$ y un nivel de confianza al $95 \%$, con relación 1:1; pareados por edad. Se obtuvo un total de 71 pacientes para cada grupo.

Cohorte expuesta: formada por pacientes con diabetes gestacional que cursaban con puerperio inmediato.

Cohorte no expuesta: formada por pacientes sin diabetes gestacional que cursaban con puerperio inmediato.

El muestreo fue no probabilístico por cuota para ambos grupos. En la cohorte expuesta, se incluyeron a las puérperas con producto único, que aceptaron participar en el estudio previo consentimiento informado y firmado; en la cohorte no expuesta a pacientes puérperas sin hábitos tóxicos, que hayan cursado con embarazo fisiológico y con producto único. En ambos grupos se excluyeron a pacientes con diabetes pregestacional tipo $1 \circ 2$, con antecedente de enfermedades crónicas, pacientes con mortinatos y de ingesta de medicamentos que pudieran afectar el desarrollo fetal.

Análisis estadístico: Los datos obtenidos se procesaron en el programa SPSS (versión 17). En el análisis estadístico se utilizó estadística descriptiva e inferencial: medias, desviación estándar, frecuencias, porcentajes; así como la prueba de Chi cuadrado y riesgo relativo con un IC del $95 \%$. 
Diagnóstico de diabetes gestacional: En México se establece el diagnóstico de diabetes gestacional según la Norma Oficial Mexicana de 1995, si durante las semanas 24 a 28 del embarazo se presentan dos o más de los siguientes valores: en ayuno $>105 \mathrm{mg} / \mathrm{dl}$ y después de una carga de glucosa en ayuno de $100 \mathrm{~g}$, valores superiores a $190 \mathrm{mg} / \mathrm{dl}$ a la hora poscarga, $165 \mathrm{mg} / \mathrm{dl}$ a las dos horas poscarga y $145 \mathrm{mg} / \mathrm{dl}$ a las tres horas.

En relación a las pacientes embarazadas sin diagnóstico de diabetes gestacional, se corroboró que la glucemia sérica estuviera dentro de parámetros normales, a través del expediente clínico. Para la obtención de la información de las gestantes se elaboró un cuestionario que incluyó variables sociodemográficas (edad y escolaridad), ginecobstétricas (antecedente de cesárea, de productos macrosómicos, de malformaciones congénitas, de obesidad y de sobrepeso pregestacional) y del embarazo actual (vía de terminación de parto). Para la obtención del nivel socioeconómico de las gestantes se utilizó el cuestionario de Graffar. Para el cálculo del índice de masa corporal (IMC) pregestacional de la gestante, se tomó en cuenta el peso y la estatura antes del embarazo.

Los recién nacidos hijos de madres con diabetes y sin diabetes, fueron evaluados clínicamente al nacimiento por el pediatra en turno. A los recién nacidos (RN) del grupo no expuesto que egresaron sin problemas, se les dio seguimiento a través de la vía telefónica o en su defecto del expediente clínico. A los RN con morbilidades que requirieron ser canalizados al área de cuneros o de terapia intensiva neonatal, se les dio seguimiento diario hasta su egreso o muerte, a fin de registrar los eventos. Todos los datos se recabaron de los expedientes clínicos.

Definición de variables:

Características antropométricas de los neonatos: considerándose como macrosómicos a los que tuvieran un peso igual o mayor del percentil 90 correspondiente y de bajo peso a los que tuvieron un peso menor al percentil 10 (9),

Periodo gestacional: considerándose RN de término el que completó 37 semanas o más de gestación y el de pretérmino el que tuvo más de 28 semanas pero menos de 37 ,

Las complicaciones metabólicas estudiadas fueron las siguientes:

Hipoglucemia: glucosa sérica por debajo de 40 mg/ dl (10),

Hipocalcemia: disminución del calcio sérico por debajo de $7 \mathrm{mg} / \mathrm{dl}$ (11),

Hipomagnesemia: magnesio sérico menor a 1,5 $\mathrm{mg} / \mathrm{dll}(12)$,
Hiperbilirrubinemia: bilirrubina indirecta de más de $13 \mathrm{mg} / \mathrm{dl}$ después de las primeras 24 horas de nacimiento o más de $10 \mathrm{mg} / \mathrm{dl}$ durante las primeras 24 horas en ausencia de hemólisis activa secundaria a incompatibilidad a grupo, sepsis u otras causas identificables (13).

Las complicaciones respiratorias fueron:

Asfixia perinatal: definida por un Apgar a los $5 \mathrm{mi}$ nutos $\leq 6$ y datos de repercusión multisistémica, Enfermedad de membrana hialina (EMH): identificada a través de hallazgos clínicos (dificultad respiratoria, taquipnea, aleteo nasal, tiraje intercostal, retracción xifoidea y el característico quejido respiratorio) y paraclínicos (radiografía de tórax y gasometría arterial),

Taquipnea transitoria del recién nacido (TTRN): definida como dificultad respiratoria que inicia poco después del nacimiento y persiste 12 a 24 horas después, debido al retardo anormal en la absorción del líquido alveolar.

Complicaciones estructurales:

Malformaciones congénitas ( $M C$ ): considerándose como un grupo de alteraciones en el desarrollo fetal que actúan antes, durante o después de la concepción, ocasionando alteración funcional o estética que requiera cirugía o limite el bienestar, siendo identificada mediante hallazgos clínicos sugestivos y en su caso a través de ecografía para la confirmación de dicho diagnóstico,

Muerte neonatal: considerada como la muerte del RN desde el momento de su nacimiento, hasta antes del séptimo día de vida extrauterina (VEU).

El presente estudio consideró la declaración de Helsinki y las recomendaciones para la investigación biomédica en seres humanos, la cual se adaptó en la 18a Asamblea Médica Mundial en la declaración de Helsinki en 1964. Revisada por la 29a Asamblea Médica Mundial en Tokio en 1975. Conforme a la norma oficial de investigación, se sujetó a su reglamentación ética y solo se requirió del consentimiento informado de los sujetos encuestados para realizar el estudio, garantizándose la confiabilidad de los resultados, sin verse afectados alguno de los entrevistados, así como la utilización de los mismos para el cumplimiento de los objetivos propuestos en el estudio. El protocolo de investigación fue revisado y aprobado por el comité de investigación de la Facultad de Medicina de la Universidad Autónoma de Querétaro.

\section{RESULTADOS}

De un total de 142 pacientes que concluyeron el estudio se reportaron los siguientes resultados: El promedio de edad en el grupo de expuestas, fue de 
$27,2 \pm 6,7$ años y en las no expuestas de $26,4 \pm 5,9$ años, con un rango de 15-44 años. Las características socioeconómicas de ambos grupos se presentan en la Tabla I. No hubo diferencias significativas en ninguna de las variables analizadas.

Tabla I

\section{ESTRATO SOCIOECONÓMICO DE MUJERES} CON Y SIN DIABETES GESTACIONAL

\begin{tabular}{lcc}
\hline $\begin{array}{l}\text { Estrato } \\
\text { socioeconómico }\end{array}$ & $\begin{array}{c}\text { Expuestos } \\
\mathrm{n}(\%)\end{array}$ & $\begin{array}{c}\text { No expuestos } \\
\mathrm{n}(\%)\end{array}$ \\
\hline Marginal & $0(0)$ & $0(0)$ \\
Obrero & $37(52)$ & $40(56)$ \\
Medio bajo & $28(40)$ & $28(39)$ \\
Medio alto & $6(8)$ & $3(4)$ \\
Alto & $0(0)$ & $0(0)$ \\
Total & $71(100)$ & $71(100)$ \\
\hline
\end{tabular}

De la cohorte de mujeres con diabetes, los antecedentes de macrosómicos se presentaron en $26,7 \%(n=19)$, mientras que en la no expuesta $32,3 \%(n=23)$, RR: 0,7; IC95\%: 0,68-4,20, diferencia no significativa. Los antecedentes de malformaciones congénitas se observaron en $5,6 \%(n=4)$ en el grupo expuesto y en el grupo de pacientes sin diabetes gestacional $4,2 \%(n=3)$, con RR: 1,3 ; IC95\%: 0,21-6,26; diferencia no significativa. El antecedente de cesárea predominó en el grupo de mujeres con diabetes gestacional en $23,9 \%(n=17)$ y en el grupo sin exposición en $14,1 \%(n=10)$, RR 2,2; IC95\%: 0,81-4,55; diferencia no significativa. El $53,5 \%(n=38)$ de las pacientes diabéticas tuvieron resolución del embarazo por cesárea, en comparación con el 25,4\% $(n=18)$ de las mujeres sanas, con un RR: 3,3; IC95\%: 1,66-6,89, $p<0,001$.

El sobrepeso pregestacional se encontró en $50 \%(n=35)$ en la cohorte expuesta y en la no expuesta $41 \%(n=29)$, RR: 1,4; IC 95\%: 0,72-7,23; diferencia no significativa. La obesidad pregestacional se presentó en $31 \%(n=22)$ en el grupo con diabetes gestacional y en la cohorte sin diabetes 14\% ( $n=10)$, RR: 2,7; IC 95\%: 1,18-6,32, $p=0,02$.

Al comparar la morbilidad y mortalidad neonatal entre la cohorte expuesta y no expuesta se encontró lo siguiente (Tabla II): la morbilidad neonatal general (metabólica, respiratoria, malformaciones congénitas, macrosomía, bajo peso al nacimiento, prematurez, asfixia perinatal) se presentó en $60 \%$ $(n=42)$ en el grupo expuesto y en $32 \%(n=23)$ en el no expuesto, RR: 3,2; IC95\%: 1,52-6,00, p=0,002. La morbilidad metabólica (hipoglucemia, hipocal- cemia, hipomagnesemia, hiperbilirrubinemia) en el grupo de RN expuestos, se presentaron en $19,7 \%$ $(n=14)$, mientras que en el grupo sin diabetes sólo 2,8\% ( $n=2)$, con un RR: 10; IC95\%: 2,40-49,05), $\mathrm{p}<0,001$. La morbilidad respiratoria (taquipnea transitoria del recién nacido, enfermedad de membrana hialina) se observó en $31,0 \%(n=22)$ en la cohorte con exposición, mientras que en la no expuesta en 5,6\% ( $n=4)$; RR: 6,7; IC95\%: 2,43-23,21, $p<0,001$. Las malformaciones congénitas (pie equino varo, cardiopatía no especificada) se presentaron en $7,02 \%(n=5)$ en el grupo expuesto y en el no expuesto en 2,81\% ( $n=2)$, RR: 2,6; IC95\%: $0,49-$ 13,94, diferencia no significativa; la malformación congénita mas frecuente en el grupo de madres con diabetes gestacional, fue pié equino varo en $60 \%$. Se identificó hipoglucemia neonatal en el $17,2 \%$ $(n=12)$ en la cohorte expuesta, mientras que en la no expuesta en un 1,4\% $(n=1)$, RR: 14,2 ; IC $95 \%$ : 1,79-112,7, $p=0,002$. La macrosomía en el grupo de RN hijos de pacientes con diabetes, se determinó en el 19,3\% ( $n=14)$, mientras que en el grupo de neonatos de madres sanas en el 5,6\% ( $n=4)$, RR: 4,1 ; IC95\%: 1,28-13,20), $p=0,02$. El bajo peso al nacimiento se identificó en el $21,1 \%(n=15)$ de los expuestos y en los no expuestos $18,3 \%(n=13)$, RR: 1,1 ; IC95\% 0,52-2,73), diferencia no significativa. La prematurez se determinó en el 12,7\% ( $n=9)$ de los neonatos para el grupo de madres con DG y el $5,6 \%(n=4)$ en las sanas, RR: 2,4; IC95\%: 0,718,29 , diferencia no significativa. La asfixia perinatal se identificó en $4,2 \%(n=3)$, en el grupo de RN hijo de madre con $D G$ y en $1,4 \%(n=1)$ en la cohorte no expuesta, RR: 3,1; IC95\% 0,31-30,42), diferencia no significativa. No hubo muerte perinatal en los grupos estudiados.

\section{DISCUSIÓN}

Este estudio estuvo enfocado en identificar si la diabetes gestacional fue un factor de riesgo para producir morbilidad en los neonatos de embarazadas controladas en Unidad de Medicina Familiar No. 16 del Instituto Mexicano del Seguro Social.

El grupo analizado más afectado por la DG en el presente estudio, fue el de 25-39 años, con una media de 27, resultado diferente con lo reportado en la literatura mundial, en donde se refiere a la edad materna de más de 35 años, como fuerte predictor para morbilidad neonatal. En un estudio multicéntrico se observó que la incidencia incrementada de complicaciones neonatales, está fuertemente relacionada con la edad materna, en dicho estudio se presentó la DG con mayor prevalencia en el grupo de 30 a 34 años, con una media de 32,3 años (9). 
Tabla II

MORBILIDAD NEONATAL ASOCIADA A LA DIABETES GESTACIONAL

\begin{tabular}{lcccc}
\hline Morbilidad & $\begin{array}{c}\text { Expuestos } \\
\mathrm{n}(\%)\end{array}$ & $\begin{array}{c}\text { No expuestos } \\
\mathrm{n}(\%)\end{array}$ & $\mathrm{RR}$; IC95\% & Valor $\mathrm{p}$ \\
\hline Morbilidad general & $42(60,0)$ & $23(32,4)$ & 3,$2 ; 1,52-6,00$ & 0,002 \\
Morbilidad metabólica $~$ & $14(19,7)$ & $2(2,8)$ & $10 ; 2,40-49,05$ & 0,000 \\
Morbilidad respiratoria $\infty$ & $22(31,0)$ & $4(5,6)$ & 6,$7 ; 2,43-23,21$ & 0,000 \\
Malformaciones congénitas $\alpha$ & $5(7,0)$ & $2(2,8)$ & 2,$6 ; 0,49-13,94$ & $0,441^{*}$ \\
Hipoglucemia neonatal & $12(17,2)$ & $1(1,4)$ & 14,$2 ; 1,79-112,70$ & 0,002 \\
Macrosomía & $14(19,3)$ & $4(5,6)$ & 4,$1 ; 1,28-13,20$ & 0,02 \\
Bajo peso al nacer & $15(21,1)$ & $13(18,3)$ & 1,$1 ; 0,52-2,73$ & $0,833^{\star}$ \\
Prematurez & $9(12,7)$ & $4(5,6)$ & 2,$4 ; 0,71-8,29$ & $0,244^{\star}$ \\
Asfixia perinatal & $3(4,2)$ & $1(1,4)$ & 3,$1 ; 0,31-30,42$ & $0,620^{\star}$ \\
Muerte perinatal & - & - & - & - \\
\hline
\end{tabular}

$\sim$ Hipoglicemia, hipocalcemia, hipomagnesemia, hiperbilirrubinemia. $\infty$ Enfermedad membrana hialina, taquipnea transitoria del recién nacido. $\alpha$ Pie equino varo, cardiopatía no especificada. * No significativo.

Nuestro estudio revela que la mayoría de las pacientes diabéticas tuvo nivel socioeconómico bajo, sin embargo, no se observó una asociación estadísticamente significativa con el grupo no expuesto, lo que manifiesta que tanto las mujeres con DG con nivel socieconómico bajo, como las que no tienen dicha patología, muestran riesgo similar para producir morbilidad neonatal, este resultado es contrario a lo encontrado por Vibeke y cols (14), posiblemente por haber realizado una asociación entre pacientes con diabetes gestacional complicadas o no.

Con respecto a la vía de terminación del embarazo vía abdominal, en nuestra investigación se encontró que la cesárea predominó en las pacientes con diabetes gestacional; este resultado es concordante a lo reportado por Orskou y cols (15), en donde dichas tasas se elevan a casi el doble de la población general; esto pudo ser por la existencia de factores de riesgo tales como: macrosomía, trastornos del líquido amniótico y el temor persistente de muerte perinatal.

Las mujeres estudiadas en nuestro estudio con $D G$, tuvieron predominancia de obesidad pregestacional sobre las no expuestas, con asociación causal significativa, diversos autores (16) refieren que la obesidad pregestacional es factor de riesgo para una elevada tasa de macrosomías y cesáreas. Ricart y cols (17), establecieron el impacto de la presencia de DG, de la obesidad de manera aislada y de ambas entidades combinadas; también observaron que la presencia de DG sin obesidad suponía por sí misma un riesgo de macrosomía, neonato grande para edad gestacional y cesárea, pero con un riesgo menor y que la presencia de obesidad sin DG presentaba riesgos mayores.

Respecto a la morbilidad metabólica (hipocalcemia e hipomagnesemia e hiperbilirrubinemia), esta se presentó en un bajo porcentaje en este estudio, comparándose con lo reportado en la literatura mundial, ya que se muestra hasta en un $40 \%$ y $30 \%$ respectivamente (18). La información en relación a la baja frecuencia de ambas patologías metabólicas neonatales, habría que tomarla con reserva debido a que en el hospital donde se realizó el estudio no es común la búsqueda de hipomagnesemia y, por otro lado, los datos clínicos son semejantes a los de hipocalcemia.

En cuanto a la morbilidad respiratoria, destacó en mayor medida la taquipnea transitoria del recién nacido (TTRN) y por último la enfermedad de membrana hialina $(E M H)$ en el grupo expuesto. Llama la atención la elevada tasa de la TTRN, debido probablemente a que en este estudio hubo una alta tasa de cesáreas, que es un factor de riesgo para la presencia de ésta patología.

En lo que respecta a la incidencia de malformaciones congénitas asociadas a las DG comparadas con el grupo no expuesto, no existió diferencia estadísticamente significativa; esto coincide con lo reportado en la actualidad, ya que la literatura médica ha sostenido el concepto de que los embarazos complicados con 
diabetes gestacional tienen el mismo riesgo para malformaciones fetales que las mujeres no diabéticas; diferente a lo manifestado por Lazalde y cols (19), Nold y cols (20) y por Cheung y cols (21), que refieren prevalencias muy altas (80\% a $90 \%$ ); estas diferencias se deben probablemente a que las malformaciones reportadas en el presente estudio están subestimadas, debido a que una proporción considerable de las mujeres embarazadas llegan al hospital en trabajo de parto, desconociéndose la presencia de DG y por carecer de estrategias de escrutinio para identificar a pacientes con este diagnóstico.

Los recién nacidos expuestos, presentaron baja prevalencia de hipoglucemia neonatal $(17,2 \%)$, pero significativamente mayor comparados con los recién nacidos no expuestos, resultados no concordantes con lo manifestado por diversos autores internacionales (22) que reportan una incidencia de 25 a 40\%, menor aún a lo reportado por Hernández-Herrera y cols (23), que la refiere en un $76,7 \%$, probablemente debido a la vigilancia activa de las determinaciones de glucosa durante las primeras horas del nacimiento, estableciendo un diagnóstico y tratamiento adecuado y oportuno.

La macrosomía predominó en los RN expuestos, hallazgo compatible con lo reportado por Greene y Solomon (24), probablemente porque la macrosomía neonatal está muy relacionada con el uso de la insulina como tratamiento y más específicamente con las dosis y los esquemas terapéuticos utilizados. En este estudio, la utilización de insulina se dio solo en la cuarta parte de las mujeres con DG y de éstas el $80 \%$ tuvo a un RN macrosómico, probablemente por un mal control metabólico. La incidencia de la macrosomía presentada en esta investigación se considera alta, algunos investigadores la informan como menor y otros de forma similar $(25,26,27,28)$.

Los recién nacidos con peso bajo $\mathrm{p} 10$ al nacer no tuvo una diferencia estadísticamente significativa en comparación con la cohorte no expuesta, compatible con lo expresado por Scholl y cols (29), los cuales no encontraron efectos de la concentración de glucosa materna en cuanto al crecimiento fetal o en el peso al nacer. Es posible que la baja comorbilidad de las madres con DG halla contribuido a este resultado.

La prematurez asociada a la DG no mostró significancia estadística, similar con dos reportes previos $(30,31)$. La falta de asociación pudo deberse a un tamaño de muestra pequeña o a la falta de control de algunas variables maternas confundentes, como el peso y la paridad.

La asfixia perinatal presentó baja frecuencia, coincidiendo este resultado con lo reportado en la literatura mundial $(28,32)$. Cabe hacer mención de que a pesar de que se reportó una alta frecuencia de interrupciones del embarazo vía abdominal, esta probablemente no se relacionó con la baja incidencia de asfixia, hallazgo que es contrario a lo manifestado por Gainor y cols (33).

La mortalidad neonatal temprana por DG en este trabajo fue nula a pesar de la elevada morbilidad neonatal asociada, esto podría explicarse porque no hubo malformaciones congénitas que fuesen incompatibles con la vida, así como una incidencia menor de prematurez, poca comorbilidad materna asociada, más diagnóstico y tratamiento nutricional materno oportuno.

\section{BIBLIOGRAFÍA}

1. Forsbach-Sánchez G, González-Obele F, VillanuevaCuéllar MA, Taméz-Pérez HE, Rocha- Márquez J. Impacto del nuevo criterio de diabetes gestacional en la estimación de su prevalencia. Rev Invest Clin 2003;55:507-10.

2. Metzger BE, Buchanan TA, Coustan DR, de Leiva A, Dunger DB, Hadden DR, Hod M, Kitzmiller JL, Kjos SL, Oats JN, Pettitt DJ, Sacks DA, Zoupas C. Fifth International Workshop-Conference on Gestational Diabetes Mellitus. Diabetes Care 2007;30(2):251-60.

3. American Diabetes Association. Diagnosis and classification of diabetes mellitus. Diabetes Care 2006;29(1):43-8.

4. Gómez Pérez FJ. Epidemiología de la diabetes en México, en: Avances en Diabetes. Primera Edición. 1999; 38-55.

5. Buchanan TA, Xiang A, Kjos SL, Watanabe R. What is gestational diabetes? Diabetes Care 2007;30(2):10511.

6. Carrapato MR, Marcelino F. The infant of the diabetic mother. The critical development windows. Early Pregnancy 2001;5:57-8.

7. Langer O, Yogev Y, Mart O, Xenakis EM. Gestational diabetes: the consequences of not treating. Am J Obstet Gynecol 2005;192: 989-97.

8. Farrel T, Neale L, Cudy T. Congenital anomalies in the offspring of women with type 2 and gestacional diabetes. Diabet Med 2002;19:322-6.

9. Battaglia FC, Lubchenco LO. A practical classification of newborn infants by weight and gestational age. $J$ Pediat 1967;71:159-63.

10. Cornblath M, Hawdorn J, Aynsley-Green A, WardPlatt M, Schwartz R, Kalhan S. Controversies regarding definition of neonatal hypoglycemia: suggested operated threshold. Pediatrics 2000;105:1141-5.

11. Robson AM. Consideraciones generales sobre la asistencia del niño enfermo: tetania hipocalcémica, Tratado de Pediatría. Madrid, España. Editorial Interamericana-Mc Grawhill 1992; 254-6.

12. Koo WK, Tsang RC. Calcium and magnesium homeostasis. Neonatology pathophysiology and management of the newborn. Fifth edition. Philadelphia: Lippincot. 1999; 715-37. 
13. Misles MJ, Gifford K, Antle CE, Leib GR. Jaundice in the healthy newborn infant: a new approach to an old problem. Pediatrics 1988;81:505-11.

14. Vibeke A, Hidde P. Van der P, Wah Cheung N, Huxley $R$, Bauman A. Sociodemographic correlates of the increasing trend in prevalence of gestational diabetes mellitus in a large population of women between 1995 and 2005. Diabetes Care 2008;31(12):2288-93.

15. Orskou J, Henriksen TB, Kesmodel U, Secher NJ. Maternal characteristics and lifestyle factors and the risk of delivering high birth weight infants. Obstet Gynecol 2003;102(1):115-20.

16. Lu GC, Rouse DJ, DuBand M, Cliver S, Kimberlin D, Hauth JC. The effect of increasing prevalence of maternal obesity on perinatal morbidity. Am J Obstet Gynecol 2001;185:845-9.

17. Ricart W, López J, Mozas J, Pericot A, Sancho MA, González N, Basells M, Luna R, Cortázar R, Navarro P, Ramírez O, Flández B, Pallardo LF, Hernández A, Ampudia J, Fernández-Real JM, Corcoy R. Body mass index has a greater impact on pregnancy outcomes tan gestational hyperglycaemia. Diabetología 2005;48:1736-42.

18. Fan ZT, Yang HX, Gao XL, Lintu H, Sun WJ. Pregnancy outcome in gestational diabetes. Int $\mathrm{J}$ Gynaecol Obstet 2006;94(1):12-6.

19. Lazalde B, Sánchez-Urbina R, García de Alba J, Ramírez-Dueñas M. Gestational diabetes mellitus and congenital malformations. Ginecol Obstet Mex 2001;69:399-405.

20. Nold J, Georgieff M. Infant of diabetic mothers. Pediatr Clin North Am 2004;51(3):619-37.

21. Cheung N, Wasmer G, Al-Ali J. Risk factors for gestational diabetes among Asian women. Diabetes Care 2001;24(5):955-60.

22. Cornblath M, Hawdorn J, Aynsley-Green A, WardPlatt M, Schwartz R, Kalhan S. Controversies regarding definition of neonatal hypoglycemia: suggested operated threshold. Pediatrics 2000;105:1141-5.

23. Hernández-Herrera R, Forsbach-Sánchez G Castillo-Martínez N, Banda-Torres M, Alcalá-Galván G,
Taméz-Pérez H. Hipoglucemia en recién nacidos de madres con diabetes mellitus. Rev Invest Clin 2006;58(4):285-8.

24. Greene MF, Solomon KG. Gestational diabetes mellitus-time to treat. N Eng J Med 2005;352 2544-6.

25. Domenech MA, Manigot DA. Diabetes gestacional. Rev Medicina 2001;61(2):25-238.

26. Gillman MW, Rifas-Shiman S, Berkey CS, Field AE, Colditz GA. Maternal gestational diabetes birth weight, and adolescent obesity. Pediatrics 2003;111(3):221-6.

27. García L, Rendón S, Medina R, Misiora N, Torres J. El recién nacido de madre diabética. Rev Med Electron 2004;26(4):1-4.

28. Terrero-Llago A, Venzant-Massó M, Reyes Salazar I, Hechavarria-Rodríguez A. Efecto de la diabetes gestacional sobre los resultados perinatales. Medisan 2005;9(2):1-4.

29. Scholl T, Chen X, Gaughan C, Smith W. Influence of maternal glucose level on ethnic differences in birth weight and pregnancy outcome. Am J Epidemiol 2000;156(6):498-506.

30. Gunter HH, Scharf A, Hertel H, Hillemanns P, Wenzlaff $P$, Maul $H$. Perinatal morbidity in pregnancies of women with preconceptional and gestational diabetes mellitus in comparison with pregnancies of non-diabetic women. Results of the perinatal registry of Lower Saxony, Germany. Z Geburtshilfe Neonatol 2006; 210(6):200-7.

31. Shefali AK, Kavitha M, Deepa R, Mohan V. Pregnancy outcomes in pre-gestational and gestational diabetic women in comparison to non-diabetic women--A prospective study in Asian Indian mothers (CURES-35). J Assoc Physicians India 2006;54:613-8.

32. Terrero-Llago A, Venzant-Massó M, Reyes Salazar I, Hechavarria-Rodríguez A. Efecto de la diabetes gestacional sobre los resultados perinatales. Medisan 2005; 9(2): 1-4.

33. Gainor RE, Fitch C, Pollard C. Maternal diabetes and perinatal outcomes in West Virginia medicaid enrollees. W V Med J 2006;102(1):314-6. 